\section{SAT0407 RISE IN THE DIAGNOSIS OF NON-RADIOGRAPHIC FORM OF AXIAL SPONDYLOARTHRITIS IN NORTHERN ISRAEL OVER TIME}

K. Zilber, S. Ginsberg, D. Rimar, M. Rozenbaum, N. Boulman, L. Kaly, A. Awisat, N. Jaries, I. Rosner, G. Slobodin. Rheumatology, Bnai Zion Medical Center, Haifa, Israel

Background: Approach to the diagnosis of axial spondyloarthrithis (axSpA) has changed in the last decade, with the aim of diagnosing the disease in its early form.

Objectives: The objective of this study was to explore change in the diagnostic pattern of axSpA in Northern Israel over the last 15 years.

Methods: Patients with the clinical diagnosis of axSpA from six rheumatology practices affiliated with the Rheumatology Unit of the Bnai Zion Medical Center in Haifa, Israel were recruited to the study. Ankylosing Spondylitis (AS) was diagnosed in the presence of sacroilitis grade 2 or more on X-ray films; all other patients were considered as having non-radiographic axSpA. All patients were subdivided by time periods to 5 groups, and percentages of patients diagnosed in the non-radiographic stage of the disease, as well as patient demographic data were compared using exact Fisher test.

Results: One hundred twenty five patients were subdivided to 5 groups by periods of diagnoses (before 2000, 2001-2004, 2005-2008, 2009-2012, 2013-2016). Gradual increase in a proportion of patients diagnosed with non-radiographic axSpA was observed over years, with statistical significance achieved in 2013$2016(p<0.05)$ (Fig.1). Patients' gender and age distribution did not differ significantly among the groups.

Fig1. Diagnoses of ankylosing spondylitis (spotted areas) and non-radiographic axial spondyloarthritis (gray areas) in 6 rheumatology practices in Northern Israel over 2 decades.

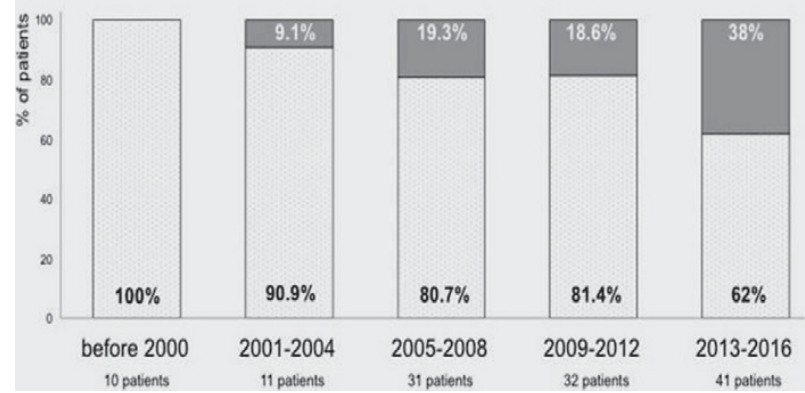

Conclusions: Progressive increase in the proportion of patients diagnosed with non-radiographic form of axSpA over years was observed in this study. This finding, made on the basis of real life data, reflects change in the diagnostic approach to spondyloarthritis during the last decades.

Disclosure of Interest: None declared

DOI: 10.1136/annrheumdis-2017-eular.5666

\section{SAT0408 POSITIVE CORRELATION BETWEEN INFLAMMATION ON SACROILIAC JOINT MRI AND SERUM C-TERMINAL TELOPEPTIDE OF TYPE-I COLLAGEN IN ANKYLOSING SPONDYLITIS BUT NOT IN NON-RADIOGRAPHIC AXIAL SPONDYLOARTHRITIS}

K.Y. Kang ${ }^{1}$, Y.S. Hong ${ }^{2}$, J.H. Ju ${ }^{3}$, S.-H. Park ${ }^{3} .{ }^{1}$ Division of Rheumatology, Internal Medicine, Catholic University of Korea, seoul; ${ }^{2}$ Catholic University of Korea, Incheon; ${ }^{3}$ Catholic University of Korea, seoul, Korea, Republic Of

Background: The MRI-determined inflammatory score was suggested as a more objective measure of disease activity than clinical activity scores in axial spondyloarthritis. It would be useful to identify the disease activity scores and/or laboratory biomarkers that objectively reflect inflammation on MRI.

Objectives: To identify the clinical disease activity scores and laboratory markers that best reflect magnetic resonance imaging (MRI)-determined sacroiliac joint (SIJ) inflammation in ankylosing spondylitis (AS) and non-radiographic axial spondyloarthritis (nr-axSpA).

Methods: This cross-sectional study included all consecutive patients who presented with axial spondyloarthritis in 2013-2015. All underwent SIJ MRI. The bone marrow edema in the inflammatory lesions on MRI was scored using the SPondyloArthritis Research Consortium of Canada (SPARCC) method. Bonespecific alkaline phosphatase (BALP), serum C-terminal telopeptide of type-I collagen (sCTX-I), and inflammatory markers were measured. Bath Ankylosing Spondylitis Disease Activity Index (BASDAI) and Ankylosing Spondylitis Disease Activity Score (ASDAS) were assessed. The correlations between the MRIdetermined SIJ inflammation scores and disease activity scores and laboratory variables were evaluated.

Results: Of the 81 patients with axSpA, 45 had AS and 36 had nr-axSpA. The AS and nr-axSpA groups did not differ in terms of disease activity scores, physical functional index, or MRI-determined SIJ inflammation. Erythrocyte sedimentation rate, C-reactive protein, and ASDAS correlated with MRI inflammatory scores in
nr-axSpA but not in AS. sCTX-I correlated with MRI-determined SIJ inflammatory scores in AS only. BASDAI and BALP levels did not associate with MRI inflammatory scores in either group. Multivariate analysis showed that $\mathrm{SCTX}-\mathrm{I}$ associated independently with MRI inflammatory score in AS ( $\beta=17.047, p=0.038)$. Conclusions: Inflammatory markers and ASDAS correlated with active sacroiliitis on MRI in nr-axSpA only. In AS, only SCTX-I correlated with active inflammation on SIJ MRI. SCTX-I may be useful as a marker of objective inflammation in AS. Disclosure of Interest: None declared

DOI: 10.1136/annrheumdis-2017-eular.1253

\section{SAT0409 IMPORTANCE OF ULTRASONOGRAPHY FOR THE DETECTION OF PERIPHERAL ENTHESITIS IN SPONDYLOARTHRITIS}

L. Mani, K. Baccouche, S. Hmila, M. Bouhoula, B. Safaa, Z. Alaya, N. El Amri, H. Zeglaoui, E. Bouajina. Rheumatology, Farhat Hached Hospital, Sousse, Tunisia

Background: Peripheral enthesitis has been repeatedly described like the primary lesion in all forms of spondyloarthritis (SpA), and it may occur at any enthesis in SpA, though it is most common in the entheses of the lower limbs. Ultrasonography (US) has proved to be a highly sensitive and noninvasive tool, to assess the presence of enthesitis

Objectives: To estimate the prevalence and the US abnormalities of enthesitis in patients with $\mathrm{SpA}$, compared to a control group.

Methods: We have consecutively included 40 patients with established SpA, including 30 patients with ankylosing spondylitis (AS), 8 patients with psoriatic arthritis (RP) and two patients with arthritis-associated with Crohn's disease. Control populations consisted of 20 patients with mechanical low back pain (MBP). Ultrasound (US) in B mode and power Doppler (PD) was performed at Achille tendon (AT) and plantar fascia (PF).

Results: The study sample included 40 control patients (4 male- 16 female) with a mean (SD) age of 51.05 (11.45) years (range 32-73) and mean duration of disease 8.40 (range 5-81) years.

SpA subtypes were: ankylosing spondylitis 30; psoriatic arthritis 8; arthritisassociated inflammatory bowel disease (AIBD) 2. 47.5\% of entheses were tender at clinical examination. Axial form was found in 36 cases $(90 \%)$ and peripheral form in 4 cases (10\%).

In SpA patients, a total of $109 / 160(68.12 \%)$ of the entheseal sites were abnormal compared with $27 / 80(33.75 \%)$ in controls, which was statistically significantly fewer than in the SpA group for AT $(p=0.000)$ and PF $(p=0.023)$. Abnormal vascularization in the cortical bone insertion was found in 47 cases $(29.375 \%)$ versus 4 cases in control group with a significant correlation. We also found a significant correlation for decreased echogenicity and bone erosions. No major variation in the proportion of abnormal entheses identified by US examination was observed between different forms of SpA.

US abnormalities were not correlated to the duration of the disease in patients with $\mathrm{SpA}$, and there was no significant difference between US findings and either ESR, CRP, BASDAI score or BASFI score. We also, found no significant correlation between entheseal US abnormalities and the anti-TNF medications used among the SpA patients.

Conclusions: Ultrasonography can be useful for detecting subclinical enthesitis in $\mathrm{SpA}$ patient and the enthesis US score can be useful for improving the diagnostic of SpA.

References:

[1] Balint PV, Kane D, Wilson H. Ultrasonography of entheseal insertions in the lower limb in spondyloarthropathy. Ann Rheum Dis 2002;61:905-10.

[2] D'Agostino MA, Said-Nahal R, Hacquard-Bouder C. Assessment of peripheral enthesitis in the spondyloarthropathies by ultrasonography combined with power Doppler: a cross-sectional study. Arthritis Rheum 2003;48:523-33.

Disclosure of Interest: None declared

DOI: 10.1136/annrheumdis-2017-eular.5230

\section{SAT0410 HLA-B27 ROLE IN ANKYLOSING SPONDYLITIS PHENOTYPE: RESULTS FROM THE REGISPONSER DATABASE}

M. Arevalo ${ }^{1}$, P. Font ${ }^{2}$, M. Moreno ${ }^{1}$, D. Ruiz ${ }^{2}$, M. Castro ${ }^{2}$, P. Carreto ${ }^{3}$

M. Larrosa ${ }^{1}$, E. Collantes ${ }^{2}$, J. Gratacós ${ }^{1}$ on behalf of REGISPONSER group.

${ }^{1}$ Rheumatology, Parc Taulí Hospital Universitari. Institut d'Investigació i Innovació

Parc Taulí I3PT. Universitat Autònoma de Barcelona. Sabadell, Spain, Sabadell;

${ }^{2}$ Rheumatology, Hospital Universitario Reina Sofía/IMIBIC/Universidad de

Córdoba., Córdoba; ${ }^{3}$ Gerency MFyC de Valladolid East Area, Valladolid, Spain

Objectives: To assess if there are different phenotypical patterns of Ankylosing Spondylitis (AS) patients depending on the positivity or negativity of HLAB27.

Methods: This is a multicentric, observational, transversal and descriptive study of AS patients from the spanish database REGISPONSER. We compared HLAB27 positive and HLAB27 negative patients regarding clinical and demographical data, disease activity and structural damage. In order to assess disease activity we used the Bath Ankylosing Spondylitis Disease Activity Index (BASDAI) and acute phase reactants (VSG and PCR). Functional disability was measured by Bath Ankylosing Spondylitis Functional Index (BASFI), and structural damage by Bath Ankylosing Spondylitis Radiology Score (BASRI). For qualitative variables we used the Chi square test and for quantitative ones the t test. An univariate 
and multivariate comparative analyisis was performed, in order to define which variables were related to the negativity or positivity of HLAB27.

Results: Data of 1235 AS pacients were analysed. Of them 1029 (83.3\%) were HLAB27 positive and 206 (17.7\%) HLAB27 negative. 924 (74.8\%) were men. AS patients with HLAB27+ presented significantly more familiar history of spondyloarhritis $(p=0.002)$, were younger $(p=0.012)$ and had earlier disease onset and disease diagnosis $(p<0.001)$, with a longer disease duration $(p=0.037)$ and a trend to a higher percentage of uveitis compared to those with HLAB27-. On the other hand, AS patients with HLAB27- also presented significantly more peripheral arthritis $(p=0.0022)$, dactylitis $(p=0.001)$ and extraarticular manifestations (psoriasis, palmoplantar pustulosis, inflammatory bowel disease (IBD) and balanitis $(p<0.001))$ compared with those of HLAB27+. AS patients with HLAB27- also presented higher scores of BASDAI and BASFI ( $p=0.047$ and $p=0.005$ respectively). The study didn't show differences between both groups of patients regarding sex distribution, axial manifestations, enthesitis nor radiological damage (assessed by $\mathrm{BASRI}$ ). In the multivariate analysis, the family history of spondyloarthritis (OR 2.10, IC95\% 1.27-3.49, p=0.004), the age at diagnosis (OR 0.97, IC95\% $0.96-0.98, \mathrm{p}<0.001$ ), the presence of dactylitis (OR 0.16, IC95\% $0.05-0.56$, $\mathrm{p}=0.004$ ), extraarticular manifestation specially IBD (OR 0.22, IC95\% $0.12-0.40$, $\mathrm{p}<0.001$ ) and peripheral arthritis (OR 0.53 , IC95\% $0.32-0.89, \mathrm{p}=0.016$ ) were the variables independently associated with the presence of HLAB27.

Conclusions: The presence of HLAB27 in AS patients is associated to an earlier disease onset, a higher frequency of familiar history of spondyloarthritis, and a lower frequency of dactylitis, extraarticular manifestations and peripheral arthritis. Disclosure of Interest: None declared

DOI: 10.1136/annrheumdis-2017-eular.1928

\section{SAT0411 CORRELATION BETWEEN DISEASE ACTIVITY SCORES AND QUALITY OF LIFE IN SPONDYLOARTHRITIS}

M. Linda, K. Baccouche, S. Lataoui, S. Belghali, Z. Alaya, N. El Amri, H. Zeglaoui, E. Bouajina. Rheumatology, Farhat Hached Hospital, Sousse, Tunisia

Background: Spondyloarthritis ( $\mathrm{SpA}$ ) is a group of chronic inflammatory rheumatism and it is known to be one of the leading causes of disability.

Objectives: This study aimed to investigate the quality of life and the psychological disorders (depression, anxiety and insomnia) in patients with SpA

Methods: A total of 60 patients were included with the diagnosis of SpA meeting the Amor and New York modified criteria, in a prospective study. In a questionnaire, the characteristics of the disease and sociodemographic patient were collected. Also psychiatric assessment was done using the insomnia severity index score (ISI) and the Hospital Anxiety and Depression scale (HAD). In addition, patients answered to the Ankylosing Spondylitis Quality of Life (ASQoL) questionnaire and the SF-12.

Results: The sex-ratio (men/women) was 3.28 (46/14), the average age was 37.95 years (18-70). The average duration of disease progression was 11.5 years (1-30). The mean value of the Bath Ankylosing Spondylitis Disease Activity Index (BASDAl) was 5.11 and the Bath Ankylosing Spondylitis Functional Index (BASFI) was 5.22 . Uveitis was reported in $11.7 \%$ of patients, osteoporosis in $35 \%$, enthesitis in $36.7 \%$ and coxitis in $36.7 \%$. $78.3 \%$ of patients received NSIAD and $30 \%$ were treated with biotheraby. On the psychological level, $25 \%$ of patients had an anxiety, $20 \%$ had depression. According to the ISI, $35 \%$ of patients had sub threshold insomnia, $20 \%$ had moderate insomnia and $10 \%$ had severe insomnia. The mean value of the ASQoL was $9(0-16)$. The mean value of the physical health was $37.13(19.34-60.41)$ and for the mental health was 41.65 (14.9-60.35). A significant positive correlation was found between the disease activity and the ASQoL $(p=0.000)$, the physical health $(p=0.000)$ and the mental health $(p=0.002)$. Also, we found a significant positive correlation between the BASDAl and depression $(p=0.01)$ and insomnia $(p=0.001)$.

Conclusions: SpA is a chronic inflammatory disease that contributes to significant physical disability and decreased quality of life in a significant number of patients. The treatment of those patients must consider the improvement of quality of life, as part of a global approach.

Disclosure of Interest: None declared

DOI: 10.1136/annrheumdis-2017-eular.6394

\section{SAT0412 ASSOCIATION BETWEEN SMOKING WITH SPINAL LEVEL OF STIFFNESS AND FUNCTIONAL LIMITATION IN PATIENTS WITH AXIAL SPONDYLOARTHRITIS: RESULTS FROM THE SPANISH ATLAS}

M. Garrido-Cumbrera ${ }^{1}$, J. Chacón-García ${ }^{1}$, J. Gratacos-Masmitja ${ }^{2}$,

D. Galvez-Ruiz ${ }^{1}$, V. Navarro-Compan ${ }^{3}$, C. Blanch-Mur ${ }^{4}$, E. Collantes-Estevez ${ }^{5}$

P. Zarco-Montejo ${ }^{6}$, O. Brace ${ }^{1} .{ }^{1}$ Universidad de Sevilla, Seville; ${ }^{2} \mathrm{H}$. Parc Taulí,

Sabadell; ${ }^{3}$ H. la Paz, Madrid; ${ }^{4}$ Novartis, Barcelona; ${ }^{5}$ Universidad de Córdoba,

Cordova; ${ }^{6}$ H. Fundación Alarcón, Madrid, Spain

Background: Smoking has been associated with greater disease activity and radiographic progression in patients with Axial Spondyloarthritis (ax-SpA). In addition, radiographic damage has been linked to greater functional limitation. However, clarification is still being sought as to whether or not this association exists. Objectives: To investigate the association between smoking and both the area of spinal stiffness and functional limitation in patients with ax-SpA.
Methods: A sample of 680 patients diagnosed with ax-SpA was interviewed during 2016 as part of the Spanish Atlas, which aims to promote early referral and improve healthcare and the use of effective treatments in patients with ax-SpA. Tobacco consumption was recorded as: Smoker (62.4\%), Occasional Smoker $(8.9 \%)$ and Non-Smoker $(28.7 \%)$. Spinal stiffness was assessed in the three different vertebral areas: cervical, dorsal and lumbar.

To determine de degree of functional limitation we used a composed index which includes the sum of the degree of limitation in the 18 daily activities well established (dressing, grooming, bathing, tying shoelaces, moving around the home, stairs, getting to/out of bed, toilet, shopping, preparing meals, eating, cleaning, walking, using public transportation, going to the doctor, driving, physical exercise, sexual relations) using an ordinal variable $(0=$ none, $1=$ little, $2=$ some and $3=$ moderate). A descriptive analysis was used to compare the level of stiffness (chi-squared test) and the mean degree of limitation (Kruskal-Wallis test) in the different groups of smokers consumptions. Regression analysis was also used to assess the relation between smoking and degree of limitation (0-54).

Results: $53 \%$ were females, mean age 46 years and $77.1 \%$ were HLA-B27+ The percentage of patients with stiffness in the lumbar region was significantly higher in habitual/occasional smokers than in non-smokers $(89.0 \%, 93.8 \%$, $83.5 \%$ respectively; $p<0.01$ ) (Table). The mean degree of functional limitation increased with tobacco consumption, although this difference was not statistically significant ( $47.9 \pm 12.1$ vs. $45.1 \pm 11.5$ vs. $44.8 \pm 13.7$ respectively; $p=0.2)$. However, regression analysis showed a statistically significant correlation between smoking and functional limitation $(r=0.096 ; p=0.02)$.

Relationship between tobacco consumption and spinal stiffness levels in patients with ax-SpA

\begin{tabular}{lccccc}
\hline & Smoker & Occasional smoker & Non smoker & $P$ & $\chi^{2}$ \\
\hline Cervical stiffness & $84.2 \%$ & $77.1 \%$ & $73.1 \%$ & 0.171 & 9.044 \\
Dorsal stiffness & $76.0 \%$ & $76.6 \%$ & $72.4 \%$ & 0.408 & 6.141 \\
Lumbar stiffness & $89.0 \%$ & $93.8 \%$ & $83.5 \%$ & 0.002 & 20.518 \\
\hline
\end{tabular}

Source: Spanish Atlas.

Conclusions: Smoking in patients with ax $\mathrm{SpA}$ is associated to greater stiffness in the lumbar region, but is not related to stiffness in the cervical or dorsal regions. Additionally, smoking is associated to the degree of functional limitation in these patients.

Acknowledgements: This project has been supported by Novartis

Disclosure of Interest: None declared

DOI: 10.1136/annrheumdis-2017-eular.6519

\section{SAT0413 INFECTIOUS PROFILE IN A TUBERCULOSIS-ENDEMIC POPULATION WITH SPONDYLOARTHROPATHIES}

M.A. Alzate $^{1,1}$, F. Ramirez ${ }^{2}$, F. Vargas ${ }^{2}$, D. Hernandez-Parra ${ }^{1}$, J.C. Salazar-Uribe ${ }^{3}$, P. Ortiz-Salazar ${ }^{1}$, R. Pineda ${ }^{1}$ on behalf of Artmedica IPS, Medellin, Colombia. ${ }^{1}$ Clinical information group; ${ }^{2}$ Rheumatology division, Artmedica IPS; ${ }^{3}$ School of Statistics, Faculty of Sciences, National University of Colombia, Medellin, Colombia

Background: Screening latent tuberculosis (TB) and other opportunistic infections in patients with spondyloarthropathies ( $\mathrm{SpA}$ ) with biologic or immunosuppression therapy is important in highly endemic population (1).

Objectives: To assess the prevalence of latent and active TB, hepatitis $B$, hepatitis C, HIV and Syphilis in a colombian cohort with SpA, as well as evaluate differences between treatments.

Methods: A cross-sectional study was conducted in 621 patients with SpAs, in whom TB, hepatitis B, hepatitis C, HIV and syphilis screening was analyzed based on type of diagnosis. Differences among immunomodulatory therapies were assessed. Statistical association was examined by means of Chi-square tests, Mann-Whitney test, and logistic regression analyses.

Results: The prevalence of latent and active TB in this cohort were $63,1 \%$, and $2,9 \%$, respectively. Significant differences were found in proportions of latent and active TB among types of SpAs, indicating a positive association with AS. A high

Table 1. Infectious profile in colombian patients with SpAs

\begin{tabular}{|c|c|c|c|c|c|c|c|c|c|}
\hline & \multicolumn{2}{|c|}{$\begin{array}{c}\text { All } \\
\mathrm{N}=621\end{array}$} & \multicolumn{2}{|c|}{$\begin{array}{c}\text { AS } \\
N=340\end{array}$} & \multicolumn{2}{|c|}{$\begin{array}{c}\mathrm{PsA} \\
\mathrm{N}=222\end{array}$} & \multicolumn{2}{|c|}{$\begin{array}{l}\text { Und SpA } \\
\mathrm{N}=59\end{array}$} & \multirow[t]{2}{*}{$\mathrm{p}$-value } \\
\hline & $\mathrm{N}$ & $\%$ & $\mathrm{~N}$ & $\%$ & $\mathrm{~N}$ & $\%$ & $\mathrm{~N}$ & $\%$ & \\
\hline Latent TB & $251 / 398$ & 63,1 & $156 / 244$ & 63,9 & $76 / 126$ & 60,3 & $19 / 28$ & 67,9 & 0,009 \\
\hline Positive TST before & & & & & & & & & \\
\hline immunotherapy & $175 / 398$ & 44 & $98 / 244$ & 40,2 & $64 / 126$ & 50,8 & $13 / 28$ & 46,4 & 0,64 \\
\hline TST conversion during & & & & & & & & & \\
\hline immunotherapy & $76 / 398$ & 19,1 & $59 / 244$ & 24,2 & $13 / 126$ & 10,3 & $4 / 28$ & 14,3 & $<.0001$ \\
\hline Active TB & 18 & 2,9 & 12 & 3,5 & 4 & 1,8 & 2 & 3,4 & 0,03 \\
\hline $\begin{array}{l}\text { Latent TB conversion to } \\
\text { active TB }\end{array}$ & 7 & 1,1 & 6 & 1,8 & 1 & 0,4 & 0 & - & 0,04 \\
\hline $\begin{array}{l}\text { Active TB during } \\
\text { immunotherapy }\end{array}$ & 11 & 1,8 & 10 & 2,9 & 1 & 0,4 & 0 & - & 0.008 \\
\hline Hepatitis B & $11 / 400$ & 2,8 & $4 / 241$ & 1,7 & $6 / 128$ & 4,7 & $1 / 31$ & 3,2 & 0,32 \\
\hline Hepatitis C & $2 / 400$ & 0,5 & $2 / 241$ & 0,8 & $0 / 128$ & - & $0 / 31$ & - & 0,61 \\
\hline VDRL test & $2 / 285$ & 0,7 & $1 / 167$ & 0,6 & $1 / 94$ & 1,1 & $0 / 24$ & - & 0,38 \\
\hline HIV & $3 / 330$ & 0,9 & $2 / 197$ & 1 & $0 / 111$ & - & $1 / 22$ & 4,5 & 0,14 \\
\hline
\end{tabular}

Ankylosing spondylitis (AS), psoriatic arthritis (PsA), undifferentiated spondyloarthritis (Und SpA), tuberculin skin test (TST). 